\title{
Ceramic foam plates: a new tool for processing fresh radical prostatectomy specimens
}

\author{
Tatjana Vlajnic • Martin Oeggerli • Cyrill Rentsch • \\ Heike Püschel • Tobias Zellweger • George N. Thalmann • \\ Christian Ruiz $\cdot$ Lukas Bubendorf
}

Received: 16 April 2014 /Revised: 25 August 2014 / Accepted: 3 October 2014 / Published online: 17 October 2014

(C) Springer-Verlag Berlin Heidelberg 2014

\begin{abstract}
Procurement of fresh tissue of prostate cancer is critical for biobanking and generation of xenograft models as an important preclinical step towards new therapeutic strategies in advanced prostate cancer. However, handling of fresh radical prostatectomy specimens has been notoriously challenging given the distinctive physical properties of prostate tissue and the difficulty to identify cancer foci on gross examination. Here, we have developed a novel approach using ceramic foam plates for processing freshly cut whole mount sections from radical prostatectomy specimens without compromising further diagnostic assessment. Forty-nine radical prostatectomy specimens were processed and sectioned from the apex to the base in whole mount slices. Putative carcinoma foci were morphologically verified by frozen section analysis. The fresh whole mount slices were then laid between two ceramic foam plates and fixed overnight. To test tissue preservation after this procedure, formalin-fixed and paraffin-embedded whole mount sections were stained with hematoxylin and eosin (H\&E) and analyzed by immunohistochemistry, fluorescence, and silver in situ hybridization (FISH and SISH, respectively). There were no morphological artifacts on H\&E stained whole mount sections from slices
\end{abstract}

T. Vlajnic $(\triangle) \cdot$ M. Oeggerli $\cdot$ H. Püschel $\cdot$ C. Ruiz $\cdot$ L. Bubendorf Institute of Pathology, University Hospital Basel, Schoenbeinstrasse 40, 4031 Basel, Switzerland

e-mail: tatjana.vlajnic@usb.ch

C. Rentsch • H. Püschel

Department of Urology, University Hospital Basel, Basel, Switzerland

T. Zellweger

Division of Urology, St. Claraspital, Basel, Switzerland

G. N. Thalmann

Department of Urology, University of Bern, Inselspital Bern, Bern, Switzerland that had been fixed between two plates of ceramic foam, and the histological architecture was fully retained. The quality of immunohistochemistry, FISH, and SISH was excellent. Fixing whole mount tissue slices between ceramic foam plates after frozen section examination is an excellent method for processing fresh radical prostatectomy specimens, allowing for a precise identification and collection of fresh tumor tissue without compromising further diagnostic analysis.

Keywords Prostate cancer $\cdot$ Ceramic $\cdot$ Biobank $\cdot$ Frozen tissue $\cdot$ Frozen section $\cdot$ Prostatectomy

\section{Introduction}

Understanding and investigating the underlying genetic and molecular mechanisms that promote neoplastic growth and progression in prostate cancer often requires access to high quality tumor tissue. Despite recent technical progress in molecular analysis of formalin-fixed and paraffin-embedded (FFPE) archived tissue [1, 2], FFPE tissue for research often remains second choice, whereas fresh-frozen tissue provides higher resolution in genomic profiling and is also preferred for RNA expression profiling. Importantly, harvesting fresh tumor tissue under sterile conditions is crucial for generating xenograft models as preclinical model systems.

In an ongoing global action plan initiative of the Movember Foundation, we aim at establishing a shared resource of well-annotated primary human prostate cancer xenografts through a multi-disciplinary effort [3]. Radical prostatectomy specimens represent a major source of tumor tissue for this purpose. However, it is notoriously difficult to identify prostate cancer by eye on gross examination. Therefore, histological verification of cancer tissue is critical prior to submitting tissue for successful xenografting or biobanking. In addition, dissection of the whole fresh prostate gland results in 
deformation of the whole mount tissue slices, which impairs further pathological assessment.

Thus, in order to preserve important pathological features for diagnosis and staging, such as margin status, tumor extent, and tumor grade, standardized protocols for handling and processing radical prostatectomy specimens have been established [4]. According to the guidelines of the International Society of Urological Pathology (ISUP), radical prostatectomy specimen should be cut only after full fixation followed by complete embedding.

Several methods for harvesting fresh prostate carcinoma tissue for research purposes have been proposed, but an optimal consensus approach still remains to be established.

Here, we have tested a novel approach using ceramic foam plates for processing freshly cut prostatectomy specimens without compromising further diagnostic assessment.

\section{Materials and methods}

\section{Tissue samples}

From April 2013 to February 2014, 49 radical prostatectomy specimens were freshly processed under sterile conditions for harvesting fresh tumor tissue to generate xenografts on NOG/ NSG mice, for biobanking, and for intraoperative analysis of resection margins. The prostatectomy specimens were processed at the pathology department of the University Hospital Basel immediately after surgical removal. The prostate was weighted and measured in three dimensions. Apical and dorsolateral margins were examined by frozen section analysis.

\section{Fresh tissue harvesting}

The remaining prostate gland tissue was sectioned at 5-7-mm intervals along a transverse plane perpendicular to the prostatic urethra from the apex to the base. If carcinoma was already present on the regular frozen sections of the margins, fresh tissue was collected from the corresponding area on the slice. Otherwise, several small samples from suspicious areas were morphologically verified on the fresh whole mounts by additional frozen section of small pieces from these areas. These small tissue pieces were marked with differently colored ink for precise allocation and put into one tissue holder for frozen section analysis (Fig. 1). Samples from confirmed cancer areas were then collected for xenografting and biobanking.

\section{Ceramic foam device}

After tissue collection, the whole mount slices were laid between two plates made of ceramic foam porous material (each measuring $20 \times 10 \times 2 \mathrm{~cm}$ ). The plates had been custom made by Composite Solutions AG, Bern, Switzerland (Fig. 2). The weight of each plate in dry state was $285 \mathrm{~g}$. They were completely saturated with $10 \%$ buffered formalin within $30 \mathrm{~min}$. The weight of a single plate soaked with formalin was $405 \mathrm{~g}$ (increase of $42 \%$ of volume), meaning that the absorbed volume of formalin was about $120 \mathrm{ml}$ for each plate. The two plates were bound together with mild pressure by standard plastic cable ties and put into a 4-1 $\left(4000 \mathrm{~cm}^{3}\right)$ container filled with $10 \%$ buffered formalin solution. After overnight fixation (for 16-20 h), the pinched whole mount tissue slices were embedded and further processed according to routine procedures. Formalin-fixed and paraffin-embedded (FFPE) whole mount sections were stained with hematoxylin and eosin (H\&E).

After each procedure, the ceramic foam plates were cleaned with a standard laboratory dishwasher.

Immunohistochemistry

FFPE tissue blocks were cut at $4 \mu \mathrm{m}$. To test if the procedure affected the tissue and antigen preservation, we applied a series of immunohistochemical (IHC) markers. Standard indirect immunoperoxidase procedures were used for the detection of Ki67 (clone MIB1, prediluted, DAKO, Glostrup, Denmark), androgen receptor (AR clone SP107, prediluted, Ventana Medical Systems Inc.), ERG (clone EPR3864, prediluted, Ventana Medical Systems Inc.), and PSA (clone ER-PR8, prediluted, Ventana Medical Systems Inc.). All analyses were performed on the BenchMark XT automated immunostainer using the OptiView detection system (Ventana Medical System Inc., Tuscon, AZ). Cytoplasmic (PSA) and nuclear (AR, ERG, Ki67) IHC markers were analyzed on one FFPE block of three specimens each. For this purpose, representative tumor areas from the whole mount FFPE blocks were cut out and re-embedded to the size of a regular FFPE block, encompassing both the peripheral and the central area of the resection specimen.

\section{FISH and SISH}

Two-color fluorescence in situ hybridization (FISH) was performed on slides from FFPE samples using Spectrum Orangelabeled androgen receptor (AR) and Spectrum Green-labeled $\mathrm{X}$-chromosome centromere region DNA probes (Vysis, Downer's Grove, IL). The hybridization was performed according to the manufacturer recommendations. Additionally, dual color silver in situ hybridization (INFORM ${ }^{\circledR} H E R 2$ DNA Probe and ultraView ${ }^{\mathrm{TM}}$ SISH Detection Kit, Ventana) was performed on slides from the same FFPE as for FISH according to the manufacturer's protocols for the INFORM HER2 DNA and chromosome 17 probes. 
Fig. 1 a Samples from macroscopically suspicious areas marked with differently colored ink and put into a tissue holder for frozen section analysis. $\mathbf{b}, \mathbf{c}$ Frozen section of a sample with prostatic adenocarcinoma, surface inked with green color, $\mathrm{H} \& \mathrm{E}$ (b magnification $\times 20$, c magnification $\times 100$ )

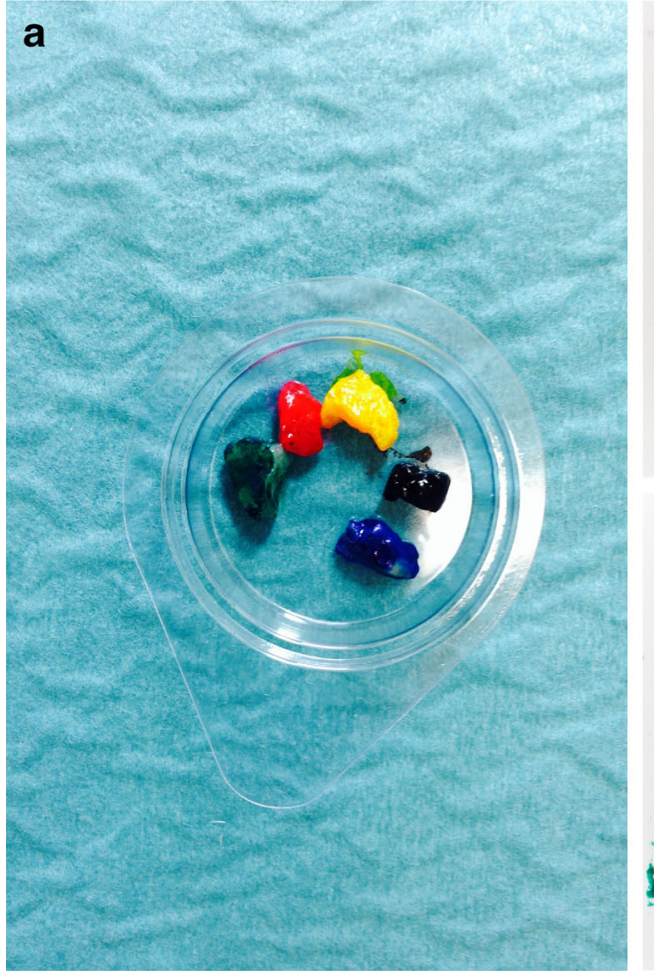

\section{Results}

We processed 49 radical prostatectomy specimens using the ceramic foam plates.

After overnight fixation, the deformation and bulging of the fresh whole tissue slices had completely disappeared and the pinched slices showed a flat surface with no artifacts caused by the mild pressure of the plates (Fig. 3).

In all 49 specimens, $H \& E$ whole mount sections revealed a normal histological architecture without irregularities, neither in the peripheral nor in the middle portions of the slide, as can

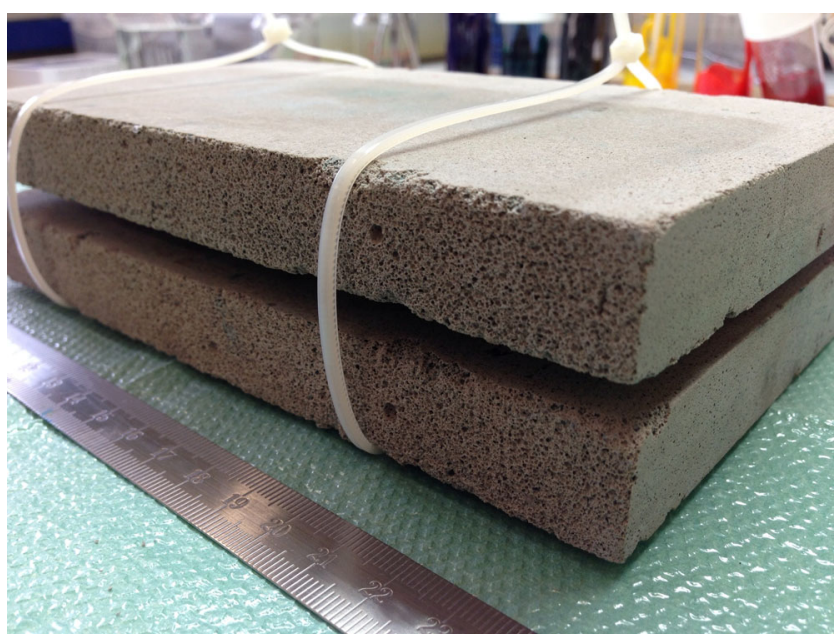

Fig. 2 Ceramic foam plates bound together by standard plastic cable ties

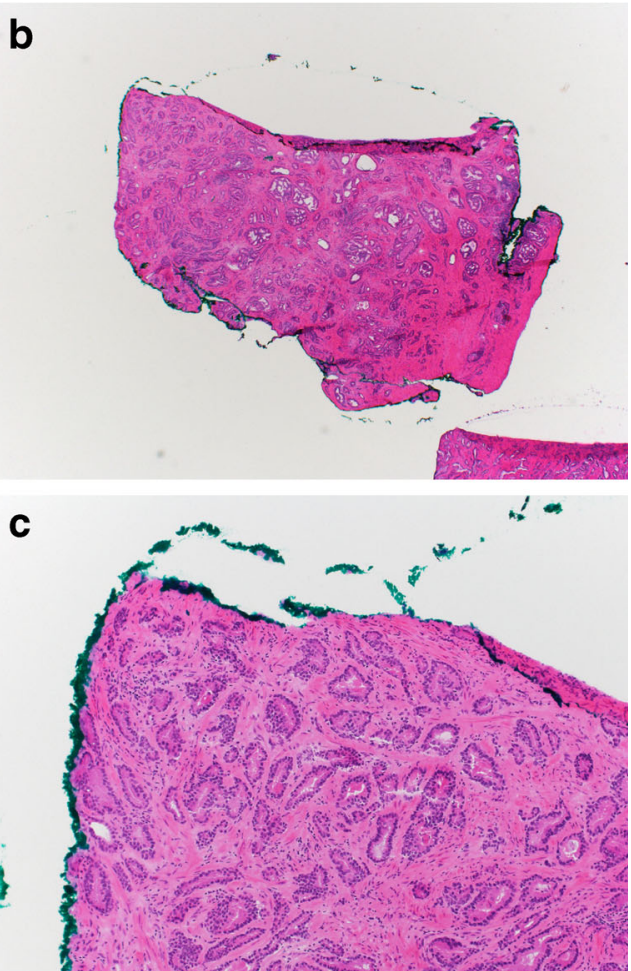

be appreciated by the well-preserved morphology of the prostatic urethra located centrally of the gland.

The IHC analysis done on three resection specimens showed homogeneous and strong expression in all ceramic foam-fixed specimens for all markers (Fig. 4). The intensity of the IHC staining was equal in the peripheral and central portions of the resection specimen.

FISH and silver in situ hybridization (SISH) signals were strong and homogeneously distributed across the slides.

\section{Discussion}

The main purpose of this study was to establish an improved method for collecting fresh tumor tissue from radical prostatectomy specimens under sterile conditions for an ongoing prostate cancer xenograft project. Here, we show that the physical properties of plates made of ceramic foam are ideal for further processing fresh whole mount slices of the prostate after fresh tissue collection.

Ceramic foam is a lightweight, typically $75-90 \%$ porous material with a three-dimensional reticulated structure [5]. It possesses high mechanical strength, chemical stability, and high temperature resistance and is therefore well suited for various applications, e.g., thermal insulation or filtration. However, its potential use as an auxiliary tool for fixation of fresh surgical specimens has not yet been evaluated. Compared to polystyrene (styrofoam) or a cork plate, ceramic foam allows for a fast penetration of formalin and therefore quick 
Fig. 3 a, b Prostate gland slices before fixation. Note the irregular cut surface. c Prostate gland slices after formalin fixation between ceramic foam plates. d Whole mount slide of prostate, $\mathrm{H} \& \mathrm{E}$
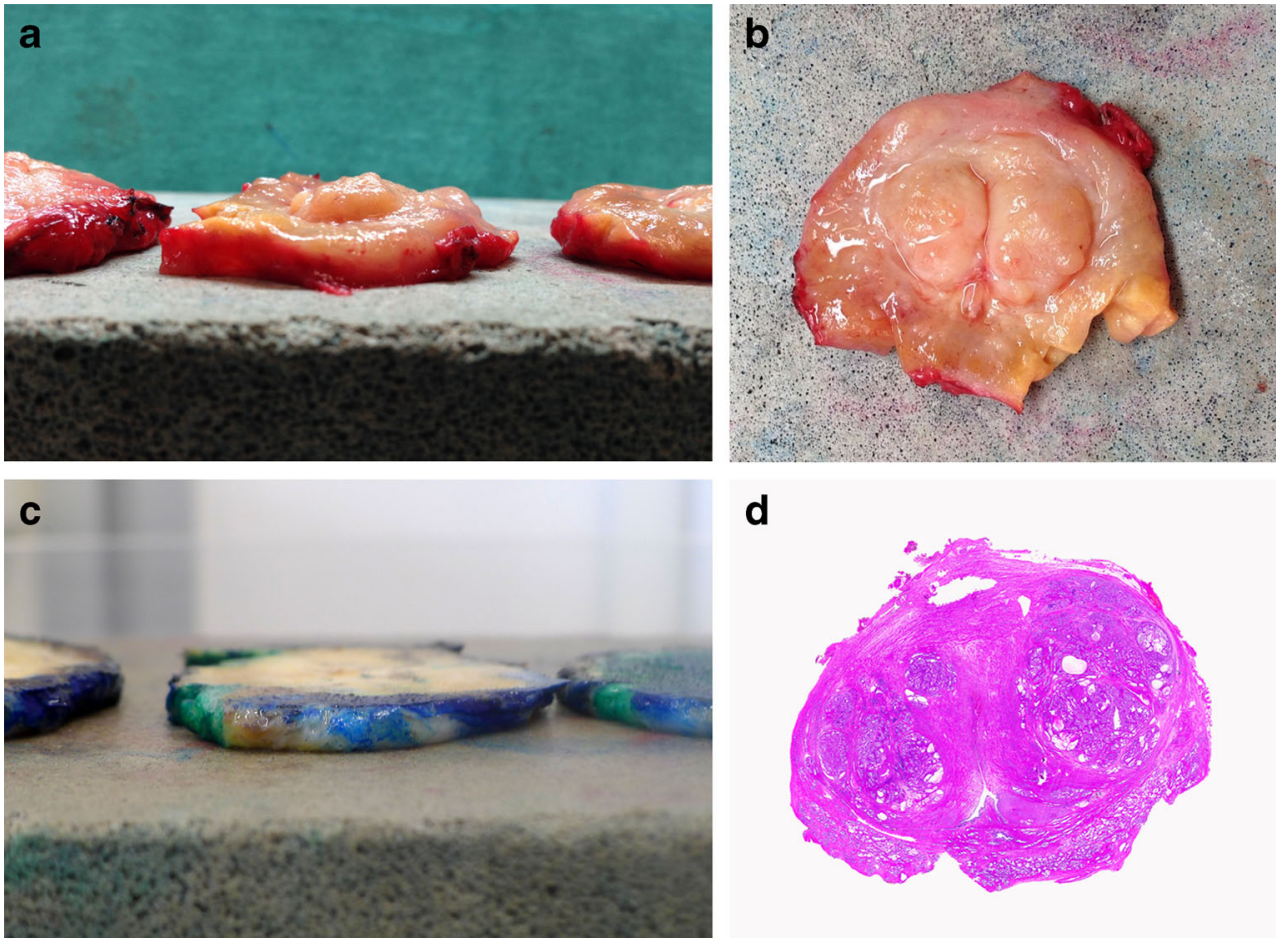

d

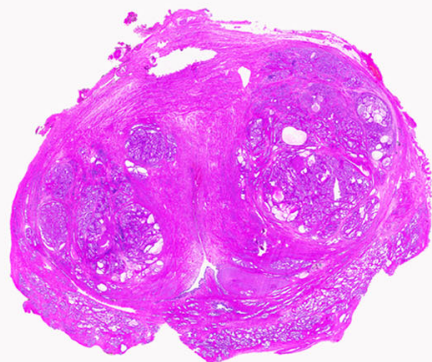

and homogeneous fixation of tissue while preventing tissue deformation. An optimal fixation of the specimen is essential for the quality of extracted DNA and for the immunohistochemical reactions. These can be compromised in large surgical specimens due to the long penetration time to the center of the specimens leading to inhomogeneous fixation and impaired tissue preservation in the central portion of the prostate [6-8]. We have shown in this study that the quality of

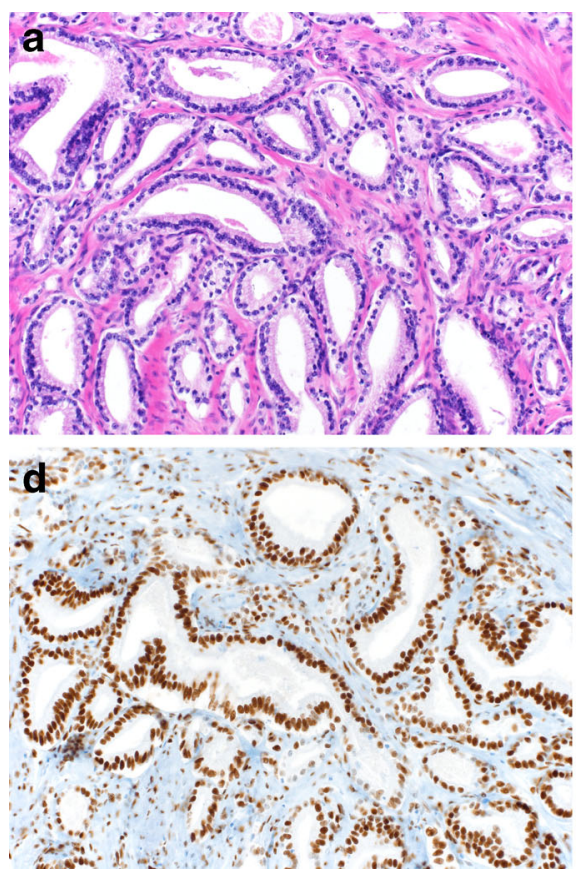

Fig. 4 a Adenocarcinoma of the prostate, H\&E. b Diffuse cytoplasmic positivity for PSA. c Negativity for ERG. Note the vascular endothelial cells as an internal positive control. d Strong and diffuse nuclear positivity for AR. e Scattered carcinoma cells with nuclear Ki67

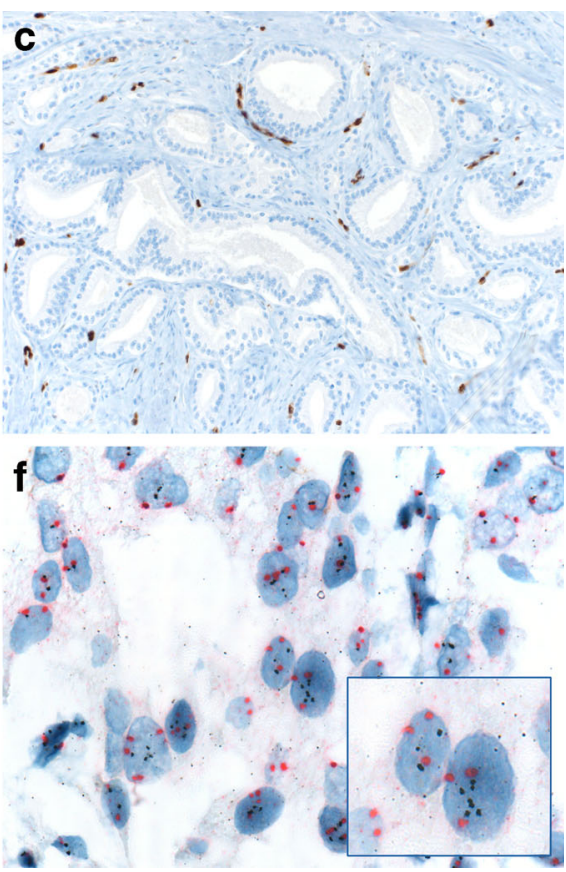

positivity (a-e magnification $\times 200$ ). f $H E R 2$ dual SISH, black HER2 signals, red CEP17 signals. Inset: two carcinoma cells with increased HER 2 copy number (magnification $\times 1000$, oil) 
the morphology as well as of the immunohistochemistry and in situ hybridization tested on specimens fixed with the ceramic foam method was excellent.

Importantly, this method does not compromise further diagnostic assessment and the morphology as well as the prostatic pseudo-capsule and the resection margins remain intact.

Several methods have previously been proposed to facilitate tumor tissue collection from fresh radical prostatectomy specimens [9-15]. Bertilsson et al. described a technique with freezing of an entire transversal slice of the prostate gland [10]. However, this method delays the process since fresh tumor tissue can only be selected after the histological workup is completed. Furthermore, it does not follow the current guidelines that recommend a complete embedding of the radical prostatectomy specimen in order not to miss important prognostic features [4]. Morrison et al. divided the central portion of every other intervening transection level into quadrants for fresh tissue procurement while removing the capsule for clinical examination [12]. The disadvantage of their method was the time required to analyze frozen section of every quadrant. Walton et al. proposed to take needle biopsies from the unfixed surgical specimen, which largely preserves the integrity of the prostate [14]. However, tumor tissue can easily been missed by such blind sampling and the amount of the harvested tissue remains small. Schäfer et al. systematically removed and snap froze inner triangles of the whole transverse sections, leaving the peripheral part of the organ intact. Although this allowed to collect tumor tissue from $>80 \%$ of the specimens without compromising further diagnostic workup, tumor tissue was randomly captured but not enriched for [13]. Warren et al. recently described a method of punching multiple (up to 27) cylindrical cores from a complete transverse slice using a large skin punch for subsequent snap freezing and storage at $-80{ }^{\circ} \mathrm{C}$ [15]. The holes on the transverse slice were inked and marked on a prostate map. After completion of the histopathology report, the frozen tissue cores were processed for frozen section analysis. This method appears to be complex and time-consuming, and the taken cores with presumable tumor are processed at a later point in time. Notably, all of these previously suggested procedures do not address the problem of tissue distortion after slicing of fresh tissue and are not suitable for immediate fresh tissue collection for xenografting.

\section{Conclusions}

Our novel method using ceramic foam plates is easily applicable and timesaving. It enables retaining the flat shape of fresh whole mount sections after collecting tumor tissue for research purposes. Therefore, this approach greatly facilitates collecting of fresh material without compromising standard diagnostic analyses.

This method could also be applied to other specimens that are often processed in unfixed state, such as resection specimens of breast cancer and soft tissue tumors.

Acknowledgments We thank Sandra Schneider and Rita Epper for excellent technical support and Manuela Bubendorf for her helpful advice.

Funding This study has partly been funded by the Movember Foundation and Astellas Pharma AG.

Disclosure The authors declare that they have no conflict of interest.

\section{References}

1. Antonov J, Goldstein DR, Oberli A, Baltzer A, Pirotta M, Fleischmann A, Altermatt HJ, Jaggi R (2005) Reliable gene expression measurements from degraded RNA by quantitative real-time PCR depend on short amplicons and a proper normalization. Lab Invest: J Tech Methods Pathol 85(8):1040-1050. doi:10.1038/ labinvest. 3700303

2. Holley T, Lenkiewicz E, Evers L, Tembe W, Ruiz C, Gsponer JR, Rentsch CA, Bubendorf L, Stapleton M, Amorese D, Legendre C, Cunliffe HE, McCullough AE, Pockaj B, Craig D, Carpten J, Von Hoff D, Iacobuzio-Donahue C, Barrett MT (2012) Deep clonal profiling of formalin fixed paraffin embedded clinical samples. PLoS One 7(11):e50586. doi:10.1371/journal.pone.0050586

3. Movember (2013) GAP1 Global Prostate Cancer Biomarker Initiative. http://ch.movember.com/en/report-cards/view/id/637/gap1-globalprostate-cancer-biomarker-initiative [accessed February 8, 2014]

4. Samaratunga H, Montironi R, True L, Epstein JI, Griffiths DF, Humphrey PA, van der Kwast T, Wheeler TM, Srigley JR, Delahunt B, Egevad L, Group IPC (2011) International Society of Urological Pathology (ISUP) consensus conference on handling and staging of radical prostatectomy specimens. Working group 1: specimen handling. Mod Pathol: Off J U S Can Acad Pathol Inc 24(1):615. doi:10.1038/modpathol.2010.178

5. Lautensack C, Giertzsch M, Godehardt M, Schladitz K (2008) Modelling a ceramic foam using locally adaptable morphology. J Microsc 230(Pt 3):396-404. doi:10.1111/j.1365-2818.2008.01998.x

6. De Marzo AM, Fedor HH, Gage WR, Rubin MA (2002) Inadequate formalin fixation decreases reliability of p27 immunohistochemical staining: probing optimal fixation time using high-density tissue microarrays. Hum Pathol 33(7):756-760

7. Leong AS, Gilham PN (1989) The effects of progressive formaldehyde fixation on the preservation of tissue antigens. Pathology 21(4): 266-268

8. Varma M, Linden MD, Amin MB (1999) Effect of formalin fixation and epitope retrieval techniques on antibody 34betaE12 immunostaining of prostatic tissues. Mod Pathol: Off J U S Can Acad Pathol Inc 12(5):472-478

9. Egevad L (2012) Handling of radical prostatectomy specimens. Histopathology 60(1):118-124. doi:10.1111/j.1365-2559.2011. 04002.x

10. Bertilsson H, Angelsen A, Viset T, Skogseth H, Tessem MB, Halgunset J (2011) A new method to provide a fresh frozen prostate slice suitable for gene expression study and MR spectroscopy. Prostate 71(5):461-469. doi:10.1002/pros.21260 
11. Jhavar SG, Fisher C, Jackson A, Reinsberg SA, Dennis N, Falconer A, Dearnaley D, Edwards SE, Edwards SM, Leach MO, Cummings C, Christmas T, Thompson A, Woodhouse C, Sandhu S, Cooper CS, Eeles RA (2005) Processing of radical prostatectomy specimens for correlation of data from histopathological, molecular biological, and radiological studies: a new whole organ technique. J Clin Pathol 58(5):504-508. doi:10.1136/jep.2004.021808

12. Morrison C, Cheney R, Johnson CS, Smith G, Mohler JL (2009) Central quadrant procurement of radical prostatectomy specimens. Prostate 69(7):770-773. doi:10.1002/pros.20925

13. Schafer SC, Pfnur M, Yerly S, Fandel TM, Jichlinski P, Lehr HA (2009) Cryopreservation of prostate cancer tissue during routine processing of fresh unfixed prostatectomy specimen: demonstration and validation of a new technique. Prostate 69(2):191-197. doi:10. 1002/pros. 20868

14. Walton TJ, McCulloch TA, Rees RC, Bishop MC (2005) Obtaining fresh prostate cancer tissue for research: a novel biopsy needle and sampling technique for radical prostatectomy specimens. Prostate 64(4):382-386. doi:10.1002/pros. 20264

15. Warren AY, Whitaker HC, Haynes B, Sangan T, McDuffus LA, Kay JD, Neal DE (2013) Method for sampling tissue for research which preserves pathological data in radical prostatectomy. Prostate 73(2): 194-202. doi:10.1002/pros.22556 